\title{
Oblivious Polynomial Evaluation and Oblivious Neural Learning
}

\author{
Yan-Cheng Chang ${ }^{1}$ and Chi-Jen Lu ${ }^{2}$ \\ 1 ROC Airforce, Taiwan r88023@csie.ntu.edu.tw \\ 2 Institute of Information Science, Academia Sinica, Taipei, Taiwan \\ cjlu@iis.sinica.edu.tw
}

\begin{abstract}
We study the problem of Oblivious Polynomial Evaluation (OPE). There are two parties, Alice who has a polynomial $P$, and Bob who has an input $x$. The goal is for Bob to compute $P(x)$ in such way that Alice learns nothing about $x$ and Bob learns only what can be inferred from $P(x)$. Previously existing protocols are based on some intractability assumptions that have not been well studied [15]14, and these protocols are only applicable for polynomials over finite fields. In this paper, we propose efficient OPE protocols which are based on Oblivious Transfer only. Unlike that of [15], slight modifications to our protocols immediately give protocols to handle multi-variate polynomials and polynomials over floating-point numbers. Many important real-world applications deal with floating-point numbers, instead of integers or arbitrary finite fields, and our protocols have the advantage of operating directly on floating-point numbers, instead of going through finite field simulation as that of [14]. As an example, we give a protocol for the problem of Oblivious Neural Learning, where one party has a neural network and the other, with some training set, wants to train the neural network in an oblivious way.
\end{abstract}

\section{Introduction}

Assume that there are two parties, Alice who has a function $f$ and Bob who has an input $x$. They want to collaborate in a way for Bob to compute $f(x)$ such that Alice learns nothing about $x$ and Bob learns only what can be inferred from $f(x)$. A protocol achieving this task for any function $f$ and any input $x$ is called an Oblivious Function Evaluation protocol. The remarkable results of Yao [17 and Goldreich, Micali, and Wigderson [9] showed that such protocols exist, under some standard cryptographic assumptions. Their protocols use a Boolean circuit to represent the function $f$ and then simulate the computation of this circuit in some oblivious way. The computational or communicational overhead of their protocols depends only linearly on the circuit size of the function $f$, which is the best one can expect from a complexity-theoretical point of view. However, their protocols are far from being practical in general, and this problem still needs a lot of work to be done. One line of research is to study cases when different representations of functions can lead to more efficient simulation.

C. Boyd (Ed.): ASIACRYPT 2001, LNCS 2248, pp. 369-384, 2001.

(C) Springer-Verlag Berlin Heidelberg 2001 
Noar and Pinkas [15] considered polynomials over finite fields. Note that any function from $m$ bits to $m$ bits can be represented by a polynomial over a finite field $G F\left(2^{m}\right)$, but its degree could go as high as $2^{m}-1$. So one would like to focus on those functions that can be represented by low degree polynomials. This turns out to have several interesting applications [158,14 12. The scheme proposed in [15] is much more efficient than the conventional way of going through oblivious circuit evaluation, but its security is based on two assumptions. One assumption is the existence of a secure Oblivious Transfer protocol while the other, proposed by themselves, is the intractability of a Noisy Polynomial Interpolation Problem. Bleichenbacher and Nguyen 3] later showed that this new assumption may be much weaker than expected and suggested the use of a possibly stronger intractability assumption on a Polynomial Reconstruction Problem. Still, no one can say how hard this problem is as it is not that well-studied. Recently, Lindell and Pinkas [14] mentioned a not-yet-published OPE protocol, which is also based on some newly proposed assumption. The assumption is that the Decisional Diffie-Hellman Assumption, denoted as DDH, also holds over the group $\mathbb{Z}_{n^{2}}^{*}$, where $n$ is the product of two large primes. Contrary to the well studied DDH over $\mathbb{Z}_{n}^{*}[2]$, more research may need to be done before one can have some confidence on this new assumption. As there may be doubt on the security of both existing OPE protocols, a more satisfactory solution is certainly welcome.

As in 1514, we will focus on the case with semi-honest parties, who may be curious but still follow the protocol. The malicious case can be handled in some standard way using commitments and zero-knowledge proofs, which will only be briefly mentioned. We will propose three OPE protocols of different flavors. Compared to previous ones, the security of our first two protocols is only based on a well-accepted cryptographic assumption, namely, the existence of a secure 1-out-of-2 oblivious transfer protocol, denoted as OT $\mathrm{OT}_{1}^{2}$. For polynomials of degree $d$ over a finite field $\mathbb{F}$, our first protocol uses $d \log |\mathbb{F}|$ invocations of $\mathrm{OT}_{1}^{2}$ while [15] needs $(2 k d+1) \log m$ invocations of $\mathrm{OT}_{1}^{2}$ for some unspecified integers $k$ and $m \gg d$ depending on their proposed assumption 11 Note that for the problem in their assumption to be intractable, at least $m$ must be very large just to prevent a brute-force algorithm that tries every possibility. So, even with their additional security concern, their protocol is better than ours only when $|\mathbb{F}|>m^{2 k}$, i.e. when $|\mathbb{F}|$ is very large. Moreover, other than carrying out OT's, our protocol involves only extremely simple computation. Our second protocol is less efficient than our first one, but we include it here as the technique for achieving security seems interesting and may have other applications. Our third protocol involves a third party who does not collude with others but may be curious, and our protocol is perfectly secure, without any cryptographic assumption. Unlike that of [15], all our protocols can immediately handle multi-variate polynomials.

One attractive feature of our protocols is that they can be modified very easily to handle floating-point numbers. This is not the case for existing OPE

\footnotetext{
${ }^{1}$ Actually they use $2 k d+1$ invocations of 1-out-of- $m$ oblivious transfer, denoted as $\mathrm{OT}_{1}^{m}$. It is known that one $\mathrm{OT}_{1}^{m}$ can be simulated by $\log m$ calls to $\mathrm{OT}_{1}^{2}$, together with several evaluations of a pseudo-random function [15].
} 
protocols which rely on some specific properties of finite fields. Many important applications in real life involve numerical computation over floating-point numbers, instead of over integers or arbitrary finite fields. There is no efficient mapping known that embeds floating-point numbers into finite fields where arithmetics can be carried out easily. The approach of [14] is to scale floating-point numbers up to integers with some book-keeping, apply some OPE protocol over integers, and then do a normalization to get back floating-point numbers. This extra work could complicate their algorithm design and slow down the performance a little. We show how our OPE protocols over finite fields can be easily modified to operate directly on floating-point numbers, and we believe that such protocols are more likely to have practical applications.

In addition to computing functions obliviously, some computational tasks may also involve security issues and people may want to perform them in some oblivious way. We use machine learning as an example, and demonstrate the applicability of our OPE protocol over floating-point numbers. Lindell and Pinkas [14] considered the scenario where two parties, each holding a private database, want to jointly construct a decision tree that classifies entries in both databases, using a so-called ID3 algorithm. Such kind of learning is not robust to changes in the sense that changes to a database may cause the whole process to be run again. We use neural network as our learning model and consider the following scenario. Alice has a neural network which is trained to some degree and she uses it to serve the classification requests from other parties. Alice wants to keep her neural network secret, while others want to keep their requests secret. This is the task of oblivious neural computing. At some point, another party Bob with a set of training examples wants to help Alice's neural network get better, maybe for his own good later. Alice wants to have a secure learning process so that Bob learns nothing from her, while Bob also wants to keep his training set secret. Later, other parties having their own training set can help Alice too, and Alice's neural network can adapt in an incremental way. This is the task of oblivious neural learning. We will apply our OPE protocol over floating-point numbers, and derive protocols for oblivious neural computing and oblivious neural learning.

The rest of the paper is organized as follows. In Section 2, we give definitions and tools that will be used later. Three OPE protocols are proposed in Section 3. We derive OPE protocols for floating-point numbers in Section 4. In Section 5 , we show oblivious protocols for neural computing and learning.

\section{Preliminaries}

For a positive integer $n$, let $[n]$ denote the set $\{1, \ldots, n\}$. For an $n$-dimensional vector $v$, let $v_{i}$, for $i \in[n]$, denote the component in the $i$ 'th dimension, and we write $v=\left(v_{1}, \ldots, v_{n}\right)=\left(v_{i}\right)_{i \in[n]}$. Fix a security parameter $\tau$, so that numbers about $2^{-\tau}$ are considered negligible and circuits of sizes about $2^{\tau}$ are considered infeasible. For a distribution $D$ over a set $S$, let $D(i)$, for $i \in S$, denote the probability of $i$ according to $D$, and define $D(A)$, for $A \subseteq S$, to be $\sum_{i \in A} D(i)$. 
Definition 1. Let $D$ and $D^{\prime}$ be two distributions over a set $S$. Their distance is defined as $d\left(D, D^{\prime}\right)=\max _{A \subseteq S} d_{A}\left(D, D^{\prime}\right)$, with $d_{A}\left(D, D^{\prime}\right)=\left|D(A)-D^{\prime}(A)\right|$.

Note that $d\left(D, D^{\prime}\right)=\frac{1}{2} \sum_{i \in S}\left|D(i)-D^{\prime}(i)\right|$, which is a useful way for calculating $d\left(D, D^{\prime}\right)$.

Definition 2. Let $D$ and $D^{\prime}$ be two distributions. They are statistically indistinguishable, denoted as $D \stackrel{s}{\equiv} D^{\prime}$, if $d\left(D, D^{\prime}\right)$ is negligible. They are computationally indistinguishable, denoted as $D \stackrel{c}{\equiv} D^{\prime}$, if $d_{A}\left(D, D^{\prime}\right)$ is negligible for any subset $A$ decided by a circuit of feasible size 2

We will assume that parties in our protocols have only circuits of feasible sizes for computation unless mentioned otherwise. So we will focus on computational security, and the default distinguishability will be the computational one.

An important cryptographic primitive is the 1-out-of-2 oblivious transfer, denoted as $\mathrm{OT}_{1}^{2}$. There are several variants which are all equivalent, and the one most suited for us is the following string version of $\mathrm{OT}_{1}^{2}$. Let $\mathbb{F}$ be a set.

Definition 3. An $O T_{1}^{2}$ protocol has two parties, Sender who has input $\left(x_{0}, x_{1}\right) \in$ $\mathbb{F}^{2}$ and Chooser who has a choice $c \in\{0,1\}$. The protocol is correct if the Chooser learns $x_{c}$ for any $\left(x_{0}, x_{1}\right)$ and $c$. The protocol is secure if both conditions below are satisfied for any $\left(x_{0}, x_{1}\right)$ and $c$ :

- Chooser cannot distinguish the distribution of Sender's messages from that induced by Sender having a different value of $x_{1-c}$.

- Sender cannot distinguish the distributions of Chooser's messages induced by $c$ and $1-c$.

Similarly one can define $\mathrm{OT}_{1}^{k}$ for any $k \geq 3$, with Sender having $k$ elements and Chooser wanting to learn one. We will use $\mathrm{OT}_{1}^{k}$, for $k \geq 2$, to denote an assumed correct and secure $\mathrm{OT}_{1}^{k}$ protocol. It is known that the existence of $\mathrm{OT}_{1}^{2}$ implies the existence of $\mathrm{OT}_{1}^{k}$ for any $k \geq 3[5[15]$.

Definition 4. A protocol for oblivious polynomial evaluation has two parties, Alice who has a polynomial $P$ over some finite field $\mathbb{F}$ and Bob who has an input $x_{*} \in \mathbb{F}$. An OPE protocol is correct if Bob learns $P\left(x_{*}\right)$ for any $x_{*}$ and $P$. It is secure if both conditions below are satisfied for any $x_{*}$ and $P$ :

- Alice cannot distinguish the distribution of Bob's messages from that induced by Bob having a different $x_{*}^{\prime}$.

- Bob cannot distinguish the distribution of Alice's messages from that induced by Alice having a different $P^{\prime}$ with $P^{\prime}\left(x_{*}\right)=P\left(x_{*}\right)$.

We say that a party in a protocol is semi-honest if the party follows the protocol but may try to learn more information than he or she should. We only focus on semi-honest parties in this paper. The case of malicious parties can

\footnotetext{
${ }^{2}$ Note that for $A$ decided by a circuit $C, d_{A}\left(D, D^{\prime}\right)=\mid \mathbf{P}_{x \in D}[C(x)=1]-$ $\mathbf{P}_{x \in D^{\prime}}[C(x)=1] \mid$.
} 
be handled in a standard way, using commitments and zero-knowledge proofs, which will only be briefly sketched for our first protocol.

Suppose $D$ and $D^{\prime}$ are two distributions depending on distributions $E$ and $E^{\prime}$ respectively. For any possible outcome $t$ of $E$ and $E^{\prime}$, let $(D \mid E=t)$ and $\left(D^{\prime} \mid E^{\prime}=t\right)$ denote the distributions of $D$ and $D^{\prime}$ conditioned on $E=t$ and $E^{\prime}=t$ respectively. Here is a useful lemma for showing $D \stackrel{c}{\equiv} D^{\prime}$, which will be used several times in our security proofs later.

Lemma 1. $D \stackrel{c}{\equiv} D^{\prime}$ provided $E \stackrel{s}{\equiv} E^{\prime}$ and $(D \mid E=t) \stackrel{c}{\equiv}\left(D^{\prime} \mid E^{\prime}=t\right)$ for any $t$.

Proof. Let $C$ be a circuit which outputs 1 with probabilities $p$ and $p^{\prime}$ with respect to $D$ and $D^{\prime}$. Let $p_{t}$ and $p_{t}^{\prime}$ denote the corresponding probabilities with respect to $(D \mid E=t)$ and $\left(D^{\prime} \mid E^{\prime}=t\right)$. Let $q_{t}=E(t)$ and $q_{t}^{\prime}=E^{\prime}(t)$. Then

$$
\begin{aligned}
\left|p-p^{\prime}\right| & =\left|\sum_{t} q_{t} p_{t}-\sum_{t} q_{t}^{\prime} p_{t}^{\prime}\right| \\
& \leq \sum_{t}\left|q_{t} p_{t}-q_{t} p_{t}^{\prime}\right|+\sum_{t}\left|q_{t} p_{t}^{\prime}-q_{t}^{\prime} p_{t}^{\prime}\right| \\
& \leq \sum_{t} q_{t}\left|p_{t}-p_{t}^{\prime}\right|+\sum_{t}\left|q_{t}-q_{t}^{\prime}\right|
\end{aligned}
$$

So if $\sum_{t}\left|q_{t}-q_{t}^{\prime}\right|$ is negligible and each $\left|p_{t}-p_{t}^{\prime}\right|$ is negligible, then $\left|p-p^{\prime}\right|$ is negligible.

Some cases later have identical $E$ and $E^{\prime}$, and we only need to check each $\left|p_{t}-p_{t}^{\prime}\right|$.

A family $H$ of functions from $S_{1}$ to $S_{2}$ is said to satisfy a pair-wise independent property if for any distinct $\alpha, \alpha^{\prime} \in S_{1}$,

$$
\mathbf{P}_{h \in H}\left[h(\alpha)=h\left(\alpha^{\prime}\right)\right]=\frac{1}{\left|S_{2}\right|} .
$$

Let $\left(H, H\left(S_{1}\right)\right)$ denote the distribution of $(h, h(v))$ with random $h \in H$ and random $v \in S_{1}$, and let $\left(H, S_{2}\right)$ denote the uniform distribution over $H \times S_{2}$. We will use the following lemma, which is a special case of the so-called Leftover Hash Lemma [1011].

Lemma 2. Let $H$ be any family of functions from $S_{1}$ to $S_{2}$ satisfying the pairwise independent property. Then $d\left(\left(H, H\left(S_{1}\right)\right),\left(H, S_{2}\right)\right) \leq \sqrt{\left|S_{2}\right| /\left|S_{1}\right|}$.

A proof of this lemma is given in the appendix for completeness.

\section{Oblivious Polynomial Evaluation Protocols}

We will present three OPE protocols of different flavors in this section. Assume that both parties have agreed that polynomials are over a finite field $\mathbb{F}$ and have degrees at most $d$. The set of such polynomials can be identified with the set $\mathbb{T}=\mathbb{F}^{d+1}$ in a natural way. Suppose now Alice has a polynomial $P(x)=$ $\sum_{i=0}^{d} a_{i} x^{i} \in \mathbb{T}$ and Bob has $x_{*} \in \mathbb{F}$. 


\subsection{The First Protocol for OPE}

To make the picture clear, we only discuss the case $\mathbb{F}=G F(p)$ for some prime $p$. The generalization to $G F\left(p^{k}\right)$ with $k>1$ is straightforward. Let $m=\left\lceil\log _{2}|\mathbb{F}|\right\rceil$. Each coefficient $a_{i}$ in the polynomial can be represented as $a_{i}=\sum_{j \in[m]} a_{i j} 2^{j-1}$ with $a_{i j} \in\{0,1\}$. For $i \in[d]$ and $j \in[m]$, let $v_{i j}=2^{j-1} x_{*}^{i}$. Note that for each $i \in[d], \sum_{j \in[m]} a_{i j} v_{i j}=a_{i} x_{*}^{i}$. The idea is to have Bob prepare $\left(v_{i j}\right)_{j \in[m]}$ and have Alice get those $v_{i j}$ with $a_{i j}=1$, in some secret way. This is achieved by having Bob prepare the pair $\left(r_{i j}, v_{i j}+r_{i j}\right)$ for a random noise $r_{i j}$, and having Alice get what she wants via $\mathrm{OT}_{1}^{2}$. Note that what Alice obtains is $a_{i j} v_{i j}+r_{i j}$. Here is our first protocol, basing only on the existence of secure $\mathrm{OT}_{1}^{2}$.

\section{Protocol 1}

1. Bob prepares $d m$ pairs $\left(r_{i j}, v_{i j}+r_{i j}\right)_{i \in[d], j \in[m]}$, with each $r_{i j}$ chosen randomly from $\mathbb{F}$.

2. For each pair $\left(r_{i j}, v_{i j}+r_{i j}\right)$, Alice runs an independent $\mathrm{OT}_{1}^{2}$ with Bob to get $r_{i j}$ if $a_{i j}=0$ and $v_{i j}+r_{i j}$ otherwise.

3. Alice sends to Bob the sum of $a_{0}$ and those $d m$ values she got. Bob subtracts $\sum_{i, j} r_{i j}$ from it to obtain $P\left(x_{*}\right)$.

Lemma 3. Protocol 1 is correct when parties are semi-honest.

Proof. The sum Bob obtains in Step 3 is $a_{0}+\sum_{i} \sum_{j}\left(a_{i j} v_{i j}+r_{i j}\right)=P\left(x_{*}\right)+$ $\sum_{i, j} r_{i j}$

Lemma 4. Protocol 1 is secure when parties are semi-honest.

Proof. First, we prove Alice's security. Suppose $P$ and $P^{\prime}$ are two distinct polynomials with $P\left(x_{*}\right)=P^{\prime}\left(x_{*}\right)=y_{*}$. According to Lemma 1 it suffices to show that for any fixed $\left(r_{i j}\right)_{i \in[d], j \in[m]}$, Alice's respective message distributions $D$ and $D^{\prime}$ induced by $P$ and $P^{\prime}$ are indistinguishable. Note that the last message from Alice is $y_{*}+\sum_{i, j} r_{i j}$ for both $P$ and $P^{\prime}$ and can be ignored. So we focus on Alice's $d m$ messages from the $d m$ independent executions of OT's. For $0 \leq k \leq d m$, let $D_{k}$ denote the distribution with the first $k$ messages from $D$ and the remaining messages from $D^{\prime}$. Assume that there exists a distinguisher $C$ for $D$ and $D^{\prime}$. A standard argument shows that $C$ can also distinguish $D_{k_{0}-1}$ and $D_{k_{0}}$ for some $k_{0}$. Note that Alice must select different elements from that pair in the $k_{0}$ 'th OT, as otherwise the two distributions are identical. Then one can break Chooser's security in $\mathrm{OT}_{1}^{2}$ when Sender has this input, because with Chooser's messages for different choices replacing the $k_{0}$ 'th message of $D_{k_{0}-1}$, we get exactly $D_{k_{0}-1}$ and $D_{k_{0}}$, which can be distinguished by $C$. As $\mathrm{OT}_{1}^{2}$ is assumed to be secure, $D$ and $D^{\prime}$ are indistinguishable, and Alice is secure.

Next, we prove Bob's security. Note that Bob sends $d m$ messages to Alice for the $d m$ independent executions of OT's. Let $x_{*} \neq x_{*}^{\prime}$, let $E$ and $E^{\prime}$ be 
Bob's respective message distributions, and let $E_{k}$ denote the distribution with the first $k$ messages from $E$ and the remaining messages from $E^{\prime}$. Suppose a distinguisher for $E$ and $E^{\prime}$ exists. Then it can also distinguish $E_{k_{0}-1}$ and $E_{k_{0}}$ for some $k_{0}$. The pairs in that $k_{0}$ 'th OT have the forms $(r, v+r)$ and $\left(r^{\prime}, v^{\prime}+r^{\prime}\right)$, for some fixed $v$ and $v^{\prime}$ and for random $r$ and $r^{\prime}$. Alice's polynomial is fixed, so which element to choose in that $k_{0}$ 'th OT is also fixed. Suppose Alice chooses the first one in that pair. Then according to Lemma 1 there is a fixed $r_{0}$ such that $E_{k_{0}-1}$ conditioned on Bob having $\left(r_{0}, v+r_{0}\right)$ and $E_{k_{0}}$ conditioned on Bob having $\left(r_{0}, v^{\prime}+r_{0}\right)$ are distinguishable. Similarly as before, one can distinguish Sender's messages when Sender has $\left(r_{0}, v+r_{0}\right)$ and $\left(r_{0}, v^{\prime}+r_{0}\right)$ respectively and Chooser selects the first element, which violates Sender's security in $\mathrm{OT}_{1}^{2}$. The case when Alice chooses the second one in that pair can be argued similarly, by noticing that the distribution $(r, v+r)$ and the distribution $(-v+r, r)$ are identical. As $\mathrm{OT}_{1}^{2}$ is assumed to be secure, so is Bob.

Theorem 1. Protocol 1 is correct and secure when parties are semi-honest.

Note that only $d m$ invocations of $\mathrm{OT}_{1}^{2}$ are required and they can be done concurrently. Also observe that if $\mathrm{OT}_{1}^{2}$ can achieve perfect security for Chooser (e.g. [1) in the information-theoretical sense, then so is Protocol 1 for Alice.

A slight modification to Protocol 1 can handle the case of malicious parties. The only complication is to enforce a malicious Bob to prepare $d m$ pairs that are consistent in the sense that there is some $x_{*}$ such that $v_{i j}=2^{j-1} x_{*}^{i}$ for every $i$ and $j$, which can be achieved as follows. Bob sends his commitments of $d m$ pairs to Alice, Alice uses $\mathrm{OT}_{1}^{2}$ to have her $d m$ choices decommitted, and Bob uses a zero-knowledge proof to convince Alice that those $d m$ pairs are consistent. All these can be done using, for example, the methods in [13].

\subsection{The Second Protocol for OPE}

The idea of our second protocol is to have Alice hide the random shares of her polynomial $P$ among other random polynomials, have Bob evaluate all of them on his input $x_{*}$, and then have Alice select those values corresponding to the shares, which sum to $P\left(x_{*}\right)$. Recall that $\mathbb{T}=\mathbb{F}^{d+1}$. Let $n=\log |\mathbb{T}|+2 \tau$. For $P \in \mathbb{T}$ and $R=\left(R_{1}, \ldots, R_{n}\right) \in \mathbb{T}^{n}$, define the function $h_{R, P}:\{0,1\}^{n} \rightarrow \mathbb{T}$ as

$$
h_{R, P}(\alpha)=P-\sum_{i \in[n]} \alpha_{i} R_{i} .
$$

It's easy to check that for any $P \in \mathbb{T}$, the class $H_{P}=\left\{h_{R, P}: R \in \mathbb{T}^{n}\right\}$ satisfies the pair-wise independent property. Here is our second OPE protocol, which is also based on $\mathrm{OT}_{1}^{2}$ only. 


\section{Protocol 2}

1. Alice generates random $R \in \mathbb{T}^{n}$ and $\alpha \in\{0,1\}^{n}$ and sends $\left(R_{1}, \ldots, R_{n}, h_{R, P}(\alpha)\right)$ to Bob. Let $R_{n+1}=h_{R, P}(\alpha)$ and $\alpha_{n+1}=1$.

2. Bob generates random $r \in \mathbb{F}^{n+1}$ and prepares $n+1$ pairs $\left(r_{i}, R_{i}\left(x_{*}\right)+\right.$ $\left.r_{i}\right)_{i \in[n+1]}$.

3. For pair $i$, Alice runs an $\mathrm{OT}_{1}^{2}$ with Bob to get $r_{i}$ if $\alpha_{i}=0$ and $R_{i}\left(x_{*}\right)+r_{i}$ otherwise.

4. Alice sends the sum of the $n+1$ values to Bob. Bob subtracts $\sum_{i=1}^{n+1} r_{i}$ from it to get $P\left(x_{*}\right)$.

Theorem 2. Protocol 2 is correct and secure when parties are semi-honest.

Proof. The correctness is obvious because the sum what Bob obtains in Step 4 is $\sum_{i=1}^{n+1} \alpha_{i} R_{i}\left(x_{*}\right)+\sum_{i=1}^{n+1} r_{i}=P\left(x_{*}\right)+\sum_{i=1}^{n+1} r_{i}$. Bob's security proof is almost identical to that of Protocol 1, so we only prove Alice's security here.

Fix any two polynomials $P, P^{\prime} \in \mathbb{T}$, let $D$ and $D^{\prime}$ denote Alice's respective message distributions, and let $E$ and $E^{\prime}$ be Alice's respective message distributions in Step 1. According to Lemma 1, it suffices to show $E \stackrel{s}{\equiv} E^{\prime}$ and $(D \mid E=t) \stackrel{c}{\equiv}\left(D^{\prime} \mid E^{\prime}=t\right)$ for each $t \in \mathbb{T}$. Using an argument similar to that in Protocol 1, one can show $(D \mid E=t) \stackrel{c}{\equiv}\left(D^{\prime} \mid E^{\prime}=t\right)$ for each $t \in \mathbb{T}$ as otherwise one can break Chooser's security in $\mathrm{OT}_{1}^{2}$. Note that the family $H_{P}$ satisfies the pairwise independent property and $E$ is the distribution $\left(H_{P}, H_{P}\left(\{0,1\}^{n}\right)\right)$. With $n=\log |\mathbb{T}|+2 \tau=(d+1) m+2 \tau$, Leftover Hash Lemma [10 11] guarantees that the distance between $E$ and the uniform distribution is at most $\sqrt{|\mathbb{T}| 2^{-n}}=2^{-\tau}$, which is negligible. Similarly $E^{\prime}$ also has a negligible distance to the uniform one. So $d\left(E, E^{\prime}\right)$ is negligible and $E \stackrel{s}{\equiv} E^{\prime}$. According to Lemma 1] Alice is secure.

Note that there are $(n+1) \log |\mathbb{T}|=O(d m(d m+\tau))$ bits sent in Step 1, $O(d m+\tau)$ executions of $\mathrm{OT}_{1}^{2}$ in Step 3, and $m$ bits sent in Step 4 .

\subsection{A Protocol for 3-Party OPE}

Here we show how to remove the use of $\mathrm{OT}_{1}^{2}$ with the help a third party Clark. As a result, our protocol does not rely on any cryptographic assumption and is information-theoretically secure when no collusion exists. Again, we assume that Alice has a polynomial $P \in \mathbb{T}$, Bob has $x_{*} \in \mathbb{F}$ and only Bob learns $P\left(x_{*}\right)$. Now the security must also hold against Clark so that the messages he receives altogether look completely random to him; i.e.,

- Clark cannot distinguish the uniform distribution from the joint distribution of messages he receives from Alice and Bob.

Note that our model is slightly different from that of Feige, Kilian, and Naor 7], who have Clark as the party to receive the result. Here is the protocol. 


\section{Protocol 3}

1. Bob sends random $\left(r_{i}\right)_{i \in[k]} \in \mathbb{F}^{k}$ to Alice. He also sends $\left(x_{i}^{\prime}=x_{*}^{i}+r_{i}\right)_{i \in[k]}$ to Clark.

2. Alice sends random $\left(s_{i}\right)_{0 \leq i \leq k} \in \mathbb{F}^{k+1}$ to Bob. She also sends $a_{0}^{\prime}=a_{0}+$ $s_{0}-\sum_{i \in[k]} a_{i} r_{i}$ and $\left(a_{i}^{\prime}=a_{i}+s_{i}\right)_{i \in[k]}$ to Clark.

3. Clark sends $y=a_{0}^{\prime}+\sum_{i \in[k]} a_{i}^{\prime} x_{i}^{\prime}$ to Bob, and Bob gets $P\left(x_{*}\right)=y-\left(s_{0}+\right.$ $\left.\sum_{i \in[k]} x_{i}^{\prime} s_{i}\right)$.

Theorem 3. Protocol 3 is correct and perfectly secure provided no collusion exists,

Proof. The correctness is easy to verify. What Alice or Clark receives is completely random. Bob receives random $\left(s_{i}\right)_{0 \leq i \leq k}$ in Step 2, and receives $P\left(x_{*}\right)+s_{0}+\sum_{i \in[k]}\left(x_{*}^{i}+r_{i}\right) s_{i}$ in Step 4, so he sees the same distribution for any polynomial $P^{\prime}$ with $P^{\prime}\left(x_{*}\right)=P\left(x_{*}\right)$. So each party is perfectly secure as long as no collusion exists.

\subsection{Generalizations}

It is not hard to see that all the protocols in this section can be easily extended to deal with multi-variate polynomials. In particular, we can solve an interesting special case: Alice has $a=\left(a_{i}\right)_{i \in[n]} \in \mathbb{F}^{n}$ while Bob has $x=\left(x_{i}\right)_{i \in[n]} \in \mathbb{F}^{n}$ and wants to learn the inner product $a \cdot x=\sum_{i \in[n]} a_{i} x_{i}$.

We have only considered the setting where Alice and Bob have their own inputs and Bob gets the final result. Later we will see a variation with each input and output shared by the two parties. We call this computing with random shares. Let's use the inner product function as an example. Suppose that Alice has $u, v \in \mathbb{F}^{n}$ and Bob has $u^{\prime}, v^{\prime} \in \mathbb{F}^{n}$. They want to compute the inner product of $u+u^{\prime}$ and $v+v^{\prime}$, and produce random shares, one for each party, that sum to the inner product. This generalization can be reduced to the original problem in the following way. Note that $\left(u+u^{\prime}\right) \cdot\left(v+v^{\prime}\right)$ is equal to

$$
(u \cdot v)+\left(u \cdot v^{\prime}+v \cdot u^{\prime}\right)+\left(u^{\prime} \cdot v^{\prime}\right)
$$

Now Alice generates a random $r \in \mathbb{F}$ and prepares the $2(n+1)$-dimensional vector

$$
a=\left(-r+u \cdot v, u_{1}, \ldots, u_{n}, v_{1}, \ldots, v_{n}, 1\right),
$$

while Bob prepares the $2(n+1)$-dimensional vector

$$
x=\left(1, v_{1}^{\prime}, \ldots, v_{n}^{\prime}, u_{1}^{\prime}, \ldots, u_{n}^{\prime}, u^{\prime} \cdot v^{\prime}\right) .
$$

Bob can obtain $a \cdot x=-r+\left(u+u^{\prime}\right) \cdot\left(v+v^{\prime}\right)$ using a protocol for the original problem, and each party now holds a random share of the inner product $\left(u+u^{\prime}\right)$. $\left(v+v^{\prime}\right)$. The variation for multi-variate polynomials can be handled similarly. 


\section{Oblivious Polynomial Evaluation for Floating-Point Numbers}

\subsection{Floating-Point Number System}

We first give the definition of a floating-point number system.

Definition 5. A floating-point number is a rational number $b= \pm \sum_{j=1}^{2 m} b_{j} 2^{m-j}$ for some $m$, with $b_{j} \in\{0,1\}$. Let $\hat{m}$ denote the floating-point number system containing all such numbers together with standard arithmetic operations.

Such a floating-point number can be represented by $2 m+1$ bits: $m$ bits for the fractional part, $m$ bits for the integral part, and 1 bit for the sign. Unlike finite fields, operations in a floating-point number system are not closed and errors may occur because of the limitation of finite precision. An underflow occurs when the produced number needs more bits for the fractional part, and a rounding takes place to convert it into the nearest number in the floating-point number system. An overflow occurs when the produced number needs more bits for the integral part, and the result is left undefined.

When we want to hide an element $v$ of a finite field $\mathbb{F}$ in our previous protocols, we generate a pair $(r, r+v)$ with a random $r \in \mathbb{F}$, so that any element of the pair itself looks completely random. There is a slight complication for floating-point numbers, but it can be easily fixed.

Lemma 5. Suppose $v, v^{\prime} \in \hat{\ell}$ for some $\ell$ and suppose $k \geq \ell+\tau+1$. The distributions of $v+r$ and $v^{\prime}+r^{\prime}$ with random $r, r^{\prime} \in \hat{k}$ have a negligible distance.

Proof. The distance is at most $\frac{\left|v-v^{\prime}\right|}{2\left(2^{k}-2^{-k}\right)+1} \leq \frac{2^{\ell+1}}{2^{k}} \leq 2^{-\tau}$.

\subsection{An OPE Protocol for Floating-Point Numbers}

Assume Alice holds $P(x)=\sum_{i=0}^{d} a_{i} x^{i}$, where $a_{i} \in \hat{m}$, and Bob holds $x_{*} \in \hat{m}$. For each $i$, let $\left|a_{i}\right|=\sum_{j=1}^{2 m} a_{i j} 2^{m-j}$, with $a_{i j} \in\{0,1\}$. All our previous protocols can be easily modified for floating-point numbers, and here we only demonstrate one, which comes from Protocol 1 . We will use $\mathrm{OT}_{1}^{3}$, which can be implemented by 2 executions of $\mathrm{OT}_{1}^{2}[15$. Let $k=(d+1) m+\tau+1$ and $n=k+\log (2 d m)$. Parties agree on the floating-point system $\hat{k}$ for random numbers, and the floating-point system $\hat{n}$ for all arithmetics so that no underflow or overflow will ever occur. Let $v_{i j}=2^{m-j} x_{*}^{i}$.

\section{Protocol 4}

1. Bob prepares $2 d m$-tuples $\left(r_{i j}, v_{i j}+r_{i j},-v_{i j}+r_{i j}\right)_{i \in[d], j \in[2 m]}$, with each $r_{i j}$ chosen randomly from $\hat{k}$.

2. For each 3 -tuple $\left(r_{i j}, v_{i j}+r_{i j},-v_{i j}+r_{i j}\right)$, Alice runs an $\mathrm{OT}_{1}^{3}$ with Bob to get $r_{i j}$ if $a_{i j}=0, v_{i j}+r_{i j}$ if $a_{i j}=1 \wedge a_{i}>0$, and $-v_{i j}+r_{i j}$ otherwise.

3. Alice sends to Bob the sum of $a_{0}$ and those $2 d m$ values she got. Bob subtracts $\sum_{i, j} r_{i j}$ from it to obtain $P\left(x_{*}\right)$. 
Note that all the arithmetic are carried out in the system $\hat{n}$, which is large enough to guarantee that no error ever occurs. Then it's not hard to verify the correctness of this protocol, while its security is guaranteed by the following.

Lemma 6. Protocol 4 is secure when parties are semi-honest.

Proof. Alice's security proof is almost identical to that of Protocol 1, so we only discuss Bob's security here. Let $x_{*}, x_{*}^{\prime} \in \hat{m}$, let $E$ and $E^{\prime}$ be Bob's respective message distributions, and let $E_{k}$ denote the distribution with the first $k$ messages from $E$ and the remaining messages from $E^{\prime}$. Suppose $E_{k_{0}-1}$ and $E_{k_{0}}$ can be distinguished, for some $k_{0}$, and the 3-tuples in that $k_{0}$ 'th OT have the forms $(r, v+r,-v+r)$ and $\left(r^{\prime}, v^{\prime}+r^{\prime},-v^{\prime}+r^{\prime}\right)$, for random $r$ and $r^{\prime}$ and for some fixed $v$ and $v^{\prime}$. Let $\ell=(d+1) m$ and note that $v, v^{\prime} \in \hat{\ell}$ because $2^{m-j} x^{i} \in \hat{\ell}$ for any $x \in \hat{m}, i \in[d]$ and $j \in[2 m]$. Then according to Lemma 5 no matter which element Alice chooses, the two distributions of that element have a negligible distance. Using Lemma 1 and adapting Bob's security proof for Protocol 1, one can show that $E$ and $E^{\prime}$ are indistinguishable.

Note that the generalizations discussed in Section 3.4 also hold for floatingpoint numbers, and we have the following theorem.

Theorem 4. Oblivious protocols exist for the problem of multi-variate polynomial evaluation (with random shares) over floating-point numbers.

\section{Oblivious Neural Learning}

\subsection{Neural Computing and Learning}

There are several variants of the neural network model. We only demonstrate our result via 2-layer feedforward neural networks with back-propagation learning. Other variants can be handled similarly.

A 2-layer feedforward neural network has an internal layer of $J$ nodes, with the $j$ 'th node having a weight vector $u_{j}=\left(u_{j 1}, \ldots, u_{j I}\right)$, and an output layer of $K$ nodes, with the $k$ 'th node having a weight vector $w_{k}=\left(w_{k 1}, \ldots, w_{k J}\right)$. Each node is associated with an activation function $f(z)=a \tanh (b z)$ (the hyperbolic tangent function). The network takes an input vector $x=\left(x_{1}, \ldots, x_{I}\right)$ and produces an output vector $o=\left(o_{1}, \ldots, o_{K}\right)$ in the following way.

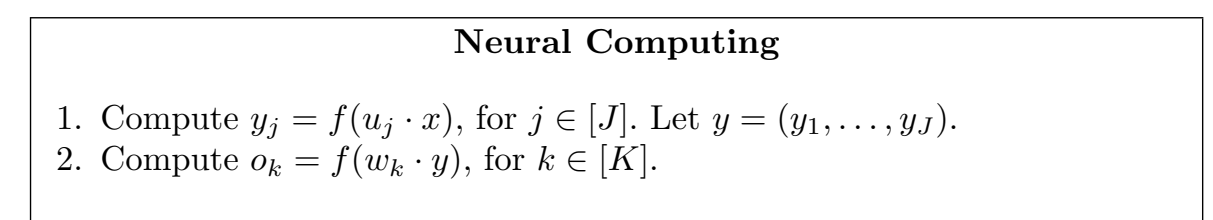

The output vector $o$ may not be correct, and a learning algorithm adjusts the weights according to how the vector $o$ differs from the correct output vector $d$. The pair $(x, d)$ constitutes a training example. The back-propagation learning (BP-Learning) algorithm adjusts the weights in the following way, with $\gamma$ being some learning constant. 


\section{BP-Learning}

1. Compute $\delta_{o k}=\frac{b}{a}\left(d_{k}-o_{k}\right)\left(a^{2}-o_{k}^{2}\right)$, for $k \in[K]$.

2. Compute $\delta_{y j}=\frac{b}{a}\left(a^{2}-y_{j}^{2}\right) \sum_{k=1}^{K} \delta_{o k} w_{k j}$, for $j \in[J]$.

3. Update $w_{k j}=w_{k j}+\gamma \delta_{o k} y_{j}$, for $k \in[K], j \in[J]$.

4. Update $u_{j i}=u_{j i}+\gamma \delta_{y j} x_{i}$, for $i \in[I], j \in[J]$.

The process above can be repeated for a set of training examples.

\subsection{Oblivious Neural Computing and Learning}

Now we want to carry out neural computing and neural learning in an oblivious way between two parties, Alice and Bob. Oblivious neural computing can be defined in a way similar to oblivious polynomial evaluation, except with Alice's polynomial replaced by a neural network. For oblivious neural learning, Bob has a set of training examples and wants to train Alice's neural network so that Bob knows nothing about Alice's neural network while Alice knows only what is implied by the weight changes. We need to be careful about Bob's security, as Alice's neural network has $I J+J K$ weights and that many weight changes may reveal a lot to Alice. So we do not let Alice know the weight changes induced by each training example, and only let her get the overall weight changes after the training of all examples. Now a learning protocol is secure for Bob if Alice cannot distinguish two training sets that give the same overall weight changes. Note in practice, neural learning typically involves large training sets.

Another scenario is for Bob to keep random shares of those final weights, as long as he is willing to help Alice serve requests from other parties for oblivious neural computing. Later when another party wants to continue the training of Alice's neural network, Bob only needs to help with his shares for the first training example, and his duty is off after that. Contrary to the previous scenario, Alice cannot learn anything about Bob's training set in this way.

\subsection{Oblivious Activation Function Evaluation}

Here we discuss options for evaluating the activation function $f(z)=$ $a \tanh (b z)=a\left(1-\frac{2}{1+e^{2 b z}}\right)$ in an oblivious way. We will rely on an protocol for oblivious circuit evaluation [17/916], denoted as OCE, which is efficient for small circuits. Assume that Alice has $x$ while Bob has $y$, and they want to generate random shares of $f(x+y)$ for Alice and Bob. One way is to use an OCE directly, if one can accept that the circuit for $f$ is reasonably small. For cases allowing a large $b, f(z)$ is close to the threshold function, which has a very simple circuit, and again we can use OCE directly. Otherwise, we will approximate $f$ in a piece-wise way by low degree polynomials and then apply our OPE protocol for it, which is described in the following. As $f$ is smooth, there are 
intervals $I_{0}=\left(-\infty, \ell_{0}\right], I_{1}=\left(\ell_{0}, \ell_{1}\right], \ldots, I_{n}=\left(\ell_{n-1}, \infty\right)$, and degree- $d$ polynomials $P_{0}, P_{1}, \ldots, P_{n}$ such that

$$
f(z) \approx P_{i}(z) \text { for } z \in I_{i},
$$

for some small $n$ and $d$, which seem good enough for practical purposes 3 Let $I$ be the function such that $I(z)=i$ for $z \in I_{i}$, which has a rather simple circuit and thus an efficient OCE protocol. Let $P_{i, x}(y)=P_{i}(x+y)$. Here is the oblivious protocol for evaluating the activation function.

\section{Protocol 5}

1. Alice generate random $r_{1}$. Bob runs OCE with Alice to get $r_{2}=I(x+$ $y)-r_{1}$.

2. Alice generate random $s_{1}$ and prepares the polynomial

$$
Q_{x}(a, y)=-s_{1}+\sum_{i=0}^{n} \frac{\prod_{j \neq i}\left(a+r_{1}-j\right)}{\prod_{j \neq i}(i-j)} P_{i, x}(y)
$$

Bob runs OPE with Alice for $s_{2}=Q_{x}\left(r_{2}, y\right)$.

Note that Alice has $s_{1}$ and Bob has $s_{2}$ with $s_{1}+s_{2}=P_{i}(x+y)$ for $x+y \in I_{i}$, so the protocol is correct. The security proof is again similar to previous ones.

\subsection{Oblivious Neural Algorithms}

First we need to determine the possible range of floating-point numbers that can ever occur during computation. Then we can determine an appropriate floatingpoint number system $\hat{k}$ for random numbers and a system $\hat{n}$ for error-free arithmetics. Here is the protocol for oblivious neural computing which uses the OCE and OPE protocols with random shares.

\section{Protocol 6}

1. For $j \in[J]$, Alice and Bob compute random shares $s_{j 1}, s_{j 2}$ of the inner product $u_{j} \cdot x$, and then compute random shares $y_{j 1}, y_{j 2}$ of $y_{j}=f\left(s_{j 1}+\right.$ $\left.s_{j 2}\right)$. Let $y=\left(y_{1}, \ldots, y_{J}\right)$.

2. For $k \in[K]$, Alice and Bob compute random shares $t_{k 1}, t_{k 2}$ of $w_{k} \cdot y$, and then compute random shares $o_{k 1}, o_{k 2}$ of $o_{k}=f\left(t_{k 1}+t_{k 2}\right)$.

At the end, Alice can send her shares $o_{k 1}$ to Bob for him to obtain the output vector $o$. This is not needed for oblivious learning. Note that the protocol still works when the each weight vector is shared by two parties instead of owned by Alice, which is the case in oblivious learning.

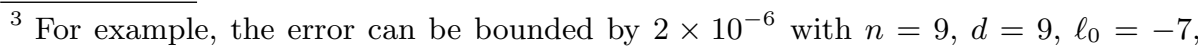
$\ell_{8}=7, P_{0}=-1$, and $P_{9}=1$.
} 
Theorem 5. Oblivious neural computing can be achieved by Protocol 5.

Proof. The correctness is easy to verify. The security relies on the security of the protocol for oblivious polynomial evaluation with random shares and the protocol for oblivious evaluation of the activation function. Any breaking of Protocol 5's security gives a way for breaking one of the protocols which has been shown to be secure.

An oblivious neural learning protocol can be derived similarly. Now only the protocol for OPE with random shares is needed.

\section{Protocol 7}

1. Alice and Bob compute random shares of each $\delta_{o k}=\frac{b}{a}\left(d_{k}-o_{k}\right)\left(a^{2}-o_{k}^{2}\right)$.

2. Alice and Bob compute random shares of each $\delta_{y j}=\frac{b}{a}\left(a^{2}-\right.$ $\left.y_{j}^{2}\right) \sum_{k=1}^{K} \delta_{o k} w_{k j}$.

3. Alice and Bob compute random shares of each $w_{k j}=w_{k j}+\gamma \delta_{o k} y_{j}$.

4. Alice and Bob compute random shares of each $u_{j i}=u_{j i}+\gamma \delta_{y j} x_{i}$.

The learning process can be repeated for a set of training examples. At the end of the whole process, Bob reveals his shares of those weights obtained in the last iteration, and Alice derives the resulting neural network. The correctness is easy to verify. The security can be proved similarly as before. Now Alice cannot distinguish among training sets that give the same overall weight changes. So we have the following theorem.

Theorem 6. Oblivious neural learning can be achieved by the combination of Protocol 6 and Protocol 7.

As discussed before, an alternative scenario is not to have Bob give away his final shares to Alice, but for him to help Alice for her future task. In this way, Alice only obtains random shares of her new weights after each training example, including the final one. So each training example is secure and now Alice learns nothing about Bob's training set.

Acknowledgements. We would like to thank Prof. Yuh-Dauh Lyuu for his help.

\section{References}

1. M. Bellare and S. Micali, Non-interactive oblivious transfer and applications, in: Proc. CRYPTO '89, Lecture Notes in Computer Science, Vol. 435 (Springer, 1990), pp. 547-557.

2. D. Boneh, Decision Diffie-Hellman problem, in: Proc. Algorithmic Number Theory 1998, Lecture Notes in Computer Science, Vol. 1423 (Springer, 1998), pp. 48-63. 
3. D. Bleichenbacher and P. Nguyen, Noisy polynomial interpolation and noisy chinese remaindering, in: Proc. EUROCRYPT 2000, Lecture Notes in Computer Science, Vol. 1807 (Springer, 2000), pp. 53-69.

4. G. Brassard, D. Chaum, and C. Crepeau, Minimum disclosure proofs of knowledge, Journal of Computer and System Sciences 37(2), 1988, pp. 156-189.

5. G. Brassard, C. Crepeau, and J. M. Robert, Information theoretical reductions among disclosure problems, in: Proc. 27th Ann. IEEE Symp. Foundations of Computer Science, 1986, pp. 168-173.

6. D. Chaum, C. Crepeau, and I. Damgard, Multiparty unconditionally secure protocols (extended abstract), in: Proc. 20th Ann. ACM Symp. Theory of Computing, 1988, pp. 11-19.

7. U. Feige, J. Kilian, and M. Naor, A minimal model for secure computation, in: Proc. 26th Ann. ACM Symp. Theory of Computing, 1994, pp. 554-563.

8. Niv Gilboa, Two party RSA key generation, in: Proc. CRYPTO '99, Lecture Notes in Computer Science, Vol. 1666 (Springer, 1999), pp. 116-129.

9. O. Goldreich, S. Micali, and A. Wigderson, How to play any mental game or a completeness theorem for protocols with honest majority, in: Proc. 19th Ann. ACM Symp. Theory of Computing, 1987, pp. 218-229.

10. J. Håstad, R. Impagliazzo, L. Levin, and M. Luby, Construction of a pseudorandom generator from any one-way function, SIAM Journal on Computing 28(4), 1999, pp. $1364-1396$.

11. R. Impagliazzo and D. Zuckerman, How to recycle random bits, in: Proc. 30th Ann. IEEE Symp. Foundations of Computer Science, 1989, pp. 248-253.

12. Y. Ishai and E. Kushilevitz, Randomizing polynomials: a new representation with applications to round-efficient secure computaion, in: Proc. 41st Ann. IEEE Symp. Foundations of Computer Science, 2000, pp. 294-304.

13. J. Kilian, Founding cryptography on oblivious transfer, in: Proc. 20th Ann. ACM Symp. Theory of Computing, 1988, pp. 20-31.

14. Y. Lindell and B. Pinkas, Privacy preserving data mining, in: Proc. CRYPTO 2000, Lecture Notes in Computer Science, Vol. 1880 (Springer, 2000), pp. 36-54.

15. M. Naor and B. Pinkas, Oblivious transfer and polynomial evaluation, in: Proc. 31st Ann. ACM Symp. Theory of Computing, 1999, pp. 245-254.

16. T. Sander, A. Young, and M. Yung, Non-interactive cryptocomputing for $\mathrm{NC}^{1}$, in: Proc. 40th Ann. IEEE Symp. Foundations of Computer Science, 1999, pp. 554-567.

17. A. C. Yao, How to generate and exchange secrets, in: Proc. 27th Ann. IEEE Symp. Foundations of Computer Science, 1986, pp. 162-167.

18. J. M. Zurada, Introduction to Artificial Neural Systems, PWS Publishing, 1994.

\section{A Proof of Lemma 2}

Let $\ell=|H|\left|S_{2}\right|$. From Cauchy-Schwartz, $\sum_{h, v}\left|\mathbf{P}_{g, u}[(g, g(u))=(h, v)]-1 / \ell\right|$ is at most

$$
\begin{aligned}
& \sqrt{\ell} \sqrt{\sum_{h, v}\left(\mathbf{P}_{g, u}[(g, g(u))=(h, v)]-1 / \ell\right)^{2}} \\
= & \sqrt{\ell \sum_{h, v} \mathbf{P}_{g, u}[(g, g(u))=(h, v)]^{2}-2+1}
\end{aligned}
$$




$$
\begin{aligned}
& =\sqrt{\ell \mathbf{P}_{h, h^{\prime}, u, u^{\prime}}\left[(h, h(u))=\left(h^{\prime}, h^{\prime}\left(u^{\prime}\right)\right)\right]-1} \\
& =\sqrt{\ell \mathbf{P}_{h, h^{\prime}}\left[h=h^{\prime}\right] \mathbf{P}_{h, u, u^{\prime}}\left[h(u)=h\left(u^{\prime}\right)\right]-1} \\
& \leq \sqrt{\ell|H|^{-1}\left(\mathbf{P}_{u, u^{\prime}}\left[u=u^{\prime}\right]+\mathbf{P}_{h, u, u^{\prime}}\left[h(u)=h\left(u^{\prime}\right) \mid u \neq u^{\prime}\right]\right)-1} \\
& =\sqrt{\left|S_{2}\right|\left(1 /\left|S_{1}\right|+1 /\left|S_{2}\right|\right)-1} \\
& =\sqrt{\left|S_{2}\right| /\left|S_{1}\right| .}
\end{aligned}
$$

